\title{
The Hydrogen Cold Work Peak in BCC Iron: Revisited, with First Principles Calculations and Implications for Hydrogen Embrittlement
}

\author{
Ronald Gibala $^{a}{ }^{\oplus}$, William Arthur Counts ${ }^{b}$, Christopher Wolverton ${ }^{c}$ \\ ${ }^{a}$ Department of Materials Science and Engineering, University of Michigan, MI 48109, Ann Arbor, USA \\ ${ }^{b}$ Apple Inc., 95014, Cupertino, CA, USA \\ 'Department of Materials Science and Engineering, Northwestern University, 60208, Evanston, IL, USA
}

Received: September 26, 2017; Revised: March 06, 2018; Accepted: April 25, 2018

\begin{abstract}
We examine experimental and theoretical results on the cold-work (Snoek-Köster) peak in bcc Fe due to H using density functional theory (DFT). We reaffirm that Seeger's interpretation of the $\mathrm{H}$ cold-work peak (Hcwp), involving motion of $\mathrm{H}$ with kinks on non-screw dislocations associated with the intrinsic-dislocation $\alpha$ peak, has experimental backing. Use of the solute-dragging theory of Schoeck suggests a H-mixed dislocation binding energy of $0.3 \mathrm{eV}$. The theory of Hirth, that the Hcwp involves $\mathrm{H}$-screw dislocation interaction manifested as the temperature-reduced intrinsic-dislocation $\gamma$ peak by the presence of $\mathrm{H}$, has merit in that our DFT calculations disclose a similar magnitude, 0.2 $\mathrm{eV}$, of $\mathrm{H}$-screw dislocation binding. This result offers support for models of $\mathrm{H}$-enhanced localized plasticity of $\mathrm{H}$ embrittlement. We also explore possible roles of $\mathrm{H}$-vacancy binding, shown by DFT to be characterized by a binding energy of $0.6 \mathrm{eV}$, in $\mathrm{H}$ trapping and $\mathrm{H}$ embrittlement and lesser effects of $\mathrm{H}$-solute binding involving small binding energies of $\sim 0.1 \mathrm{eV}$.
\end{abstract}

Keywords: Hydrogen in Iron, Cold Work Peak, Density Functional Theory.

\section{Background}

The cold work peak (cwp), also referred to as the SnoekKöster peak ${ }^{1,2}$, in deformed bcc metals containing interstitial solutes has been studied for many decades. Although the detailed mechanism of the cwp might be debated, it certainly involves dislocation-interstitial solute interaction. Of particular interest has been a cwp due to hydrogen (Hcwp) in deformed bcc $\mathrm{Fe}$ because of its potential relevance to the $\mathrm{H}$-induced reduction in the yield strength of $\mathrm{Fe}$ and to $\mathrm{H}$ embrittlement and stress corrosion cracking of $\mathrm{Fe}$ and steels ${ }^{3}$. The Hcwp occurs at temperatures of $\sim 100 \mathrm{~K}$ to $220 \mathrm{~K}$ in the low $\mathrm{Hz}$ to high $\mathrm{kHz}$ frequency range, making it kinetically compatible with these phenomena. The relaxation strength depends strongly upon $\mathrm{H}$ concentration and plastic deformation.

Early investigations by $\mathrm{Gibala}^{4}$, Miodownik ${ }^{5}$ and others attributed the Hcwp to viscous dragging of $\mathrm{H}$ atoms by vibrating-string-like dislocations, as described by the Schoeck solute-dragging model ${ }^{6}$, which had been intended for application to cwp's for heavy-interstitial-solute systems such as $\mathrm{Fe}-\mathrm{C}^{1,2}, \mathrm{Fe}-\mathrm{N}^{7}$ and $\mathrm{Nb}-\mathrm{N}^{8}$. Use of the Schoeck model leads to a H-dislocation binding energy of $0.3 \mathrm{eV}^{4,9}$, in agreement with other analyses of internal friction data in $\mathrm{Fe}-\mathrm{H}$ alloys involving effects of cold work and $\mathrm{H}$ outgassing ${ }^{4}$. The types of dislocations involved in such experiments were not determined, but since most experimental work utilized room-temperature deformation ${ }^{10}$, an assumption can be made that the binding energy corresponds to $\mathrm{H}$-mixed dislocation interaction. Binding energies of this magnitude have been obtained in other types of studies such as permeation, thermal desorption spectroscopy, and similar kinetic or thermodynamic investigations ${ }^{11}$.

A shortcoming of these early investigations is that they were completed before full recognition of intrinsic dislocation relaxation peaks in bcc metals discussed by Chambers ${ }^{12}$, Fantozzi and Ritchie ${ }^{13}$, and Schultz ${ }^{14}$. Briefly, all high-purity bcc metals appear to have two distinct Bordoni-like relaxation peaks ${ }^{15}$, one at cryogenic temperatures typically below 77 $\mathrm{K}$ called the $\alpha$ peak due to intrinsic motions of non-screw dislocations and a second at much higher temperatures, room temperature or above, called the $\gamma$ peak and associated with double kink formation in screw dislocations.

Seeger ${ }^{16}$ developed a model for the cwp's observed in heavy-interstitial systems and based on the formation of double kinks in screw dislocations in the presence of interstitial solute atmospheres. He argued that while the Hcwp might involve similar dislocation-solute interaction, the active dislocations had to be non-screw types intrinsically mobile at much lower temperatures than screw dislocations and associated with the $\alpha$ peak (and possibly its sub-peaks) ${ }^{13,14}$.

On the other hand, as part of a comprehensive review of $\mathrm{H}$ in bcc Fe and steels, Hirth ${ }^{3}$ associated the Hcwp with reductions in the yield stress of $\mathrm{Fe}$ and concomitantly-observed enhanced mobility of screw dislocations in the presence of $\mathrm{H}^{17}$. He interpreted the Hcwp in terms of a temperature-reduced $\gamma$ peak due to $\mathrm{H}$ and estimated a $\mathrm{H}$-screw dislocation binding energy of $0.2-0.3 \mathrm{eV}$ from the extent to which the $\gamma$ peak was apparently reduced in temperature. 
Other authors have attempted to address and reconcile the Seeger and Hirth interpretations. Gibala and Kumnick ${ }^{18}$ pointed out correlated changes in the $\alpha$ peak and the Hcwp but also recognized the possibility of a $\mathrm{H}$-induced temperature reduction of the $\gamma$ peak. Figure 1, taken from their paper, illustrates schematically how either the $\alpha$ peak or the $\gamma$ peak might result in the Hcwp with additions of $H$. In support of the Hirth interpretation, Kirchheim ${ }^{19}$ has offered comprehensive thermodynamic analyses of $\mathrm{H}$-dislocation interaction in bcc Fe and other metals and has argued that a decrease in the $\gamma$-peak temperature by $\mathrm{H}$ is consistent with his concept of defectant-controlled processes.

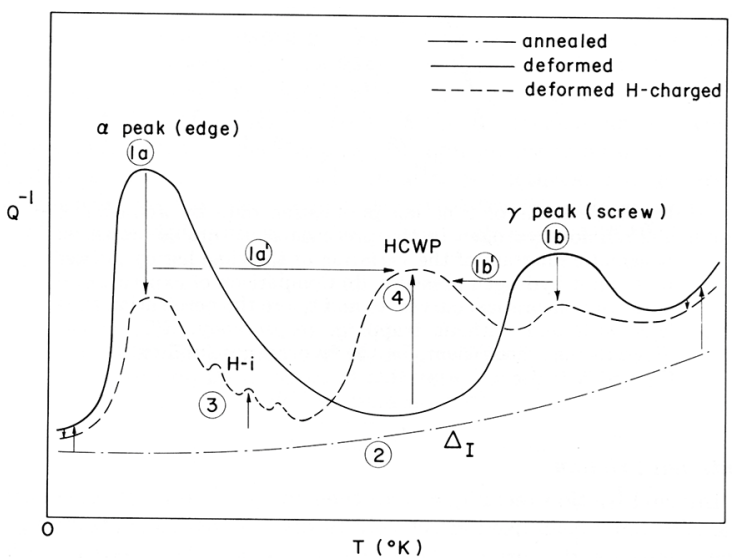

Figure 1. Schematic illustration of a possible internal friction spectrum of deformed and H-charged bcc Fe. At $1 \mathrm{~Hz}$, the $\alpha$ peak occurs at $\sim 30 \mathrm{~K}$, the Hcwp at $\sim 100-150 \mathrm{~K}$, and the $\gamma$ peak at $\sim 300$ $\mathrm{K}$. Also shown are possible $\mathrm{H}$-point defect peaks designated "H-i" at temperatures above the $\alpha$ peak (from Gibala and Kumnick ${ }^{18}$ ).

We re-examine experimental and theoretical results on $\mathrm{H}$ in bcc Fe and present additional results and interpretations based on computation of $\mathrm{H}$-defect interactions using density functional theory (DFT) methods. The H-defect interactions include ones for $\mathrm{H}$-screw dislocation, $\mathrm{H}$-vacancy, $\mathrm{H}$-solute, and $\mathrm{H}-\mathrm{H}$ binding.

\section{DFT Methodology and Results}

The first-principles calculations for H-point defect interactions (H-vacancy, $\mathrm{H}$-solute, $\mathrm{H}-\mathrm{H}$ ) are described in detail elsewhere ${ }^{20,21}$. They are based on DFT as implemented in the Vienna Ab-initio Simulation Package. The calculations are performed using the projector augmented wave potentials and the generalized gradient approximation with the exchangecorrelation functional of Perdew, Burke, and Ernzerhof. The calculations employ spin polarization to account for the ferromagnetic state of bcc Fe. They also utilize Fermi smearingof the electronic occupancy with a width of 0.05 $\mathrm{eV}$ and a plane-wave cut-off energy of $450 \mathrm{eV}$ for point defect calculations and $0.5 \mathrm{eV}$ and $400 \mathrm{eV}$, respectively, for screw dislocations.

The DFT calculations allow for atom relaxations with respect to supercell shape and volume and all atomic positions to find minimum energies and optimized defect structures. The supercell for point defect calculations is a $3 \times 3 \times 3$ cell that contains $54 \mathrm{Fe}$ atoms and serves as a reference structure for screw dislocation calculations performed in the present study.

In order to simulate a single screw dislocation, the DFT calculations additionally utilize the first-principles Green's function flexible boundary conditions ${ }^{22,23}$. This approach provides a self-consistent method to couple the dislocation strain field to the long-range elastic field of the host lattice ${ }^{24,25}$. A 540 atom computational cell was used to determine the optimized structure of the screw dislocation. It included a central core region (ccr) of 108 atoms that made up two $1 / 2<111>$ periodic units along the screw direction and for which DFT calculations were made. The ccr was imbedded annularly within two additional computational regions consisting of the remaining 432 atoms that, by the Green's function method, respectively coupled the ccr to the bulk response function and isolated it electrostatically from domain boundaries normal to $<111>$.

The optimized structure of the 108-atom, four-layer unit is given in Figure 2, for one such layer, in terms of differential displacement maps introduced by Vitek and coworkers $^{26}$. These maps identify large 'screw' components of the displacement field nominally in the direction of the dislocation line (Figure 2a) and much smaller in-plane 'edge' component displacements (Figure 2b).
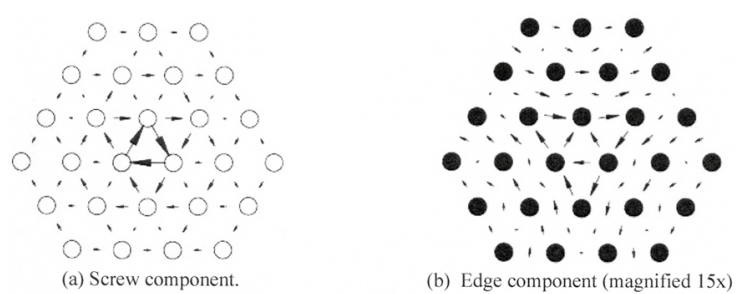

Figure 2. DFT-determined differential displacement map of the screw dislocation in bcc Fe, showing (a) the large 'screw' component displacements and (b) the much smaller 'edge' component displacements.

The energies of a single $\mathrm{H}$ atom inserted in various four-fold tetrahedral sites (t-sites), two-fold octahedral sites (o-sites), and five-fold pyramidal sites ( $p$-sites) were determined for distances extending to $3.5 \AA$ from the dislocation core. The energy landscape for $\mathrm{H}$ atoms near the dislocation core is complex. Many of the interstitial sites are unstable for $\mathrm{H}$ occupancy, in that a $\mathrm{H}$ atom placed in such sites spontaneously relaxes with no effective energy barrier to another site. All o-sites examined were found to be unstable, as were some of the t-sites and p-sites; the p-sites offered the most stable positions for H occupancy.

Within the ccr, we identify four local computational regions relative to the dislocation core: a near-core region to $0.8 \AA$; Region $\mathrm{A}$ from $0.8 \AA-1.8 \AA$; Region $\mathrm{B}$ from $1.8 \AA-3.0$ $\AA$; and Region $\mathrm{C}$ beyond $3.0 \AA$. Results for binding energies of stable $\mathrm{H}$-screw dislocation complexes are summarized in Figure 3. The largest binding energies of $0.10 \mathrm{eV}-0.17 \mathrm{eV}$ for $\mathrm{t}$-sites and $\mathrm{p}$-sites are found in or near Region $\mathrm{A}$ at distances of $1.0 \AA-2.0 \AA$ from the core. Figure 3 includes calculations 
for some unstable t-sites and $\mathrm{p}$-sites at distances beyond 2 $\AA$ and compares them for reference to the t-site and o-site energies of $\mathrm{H}$ in the dislocation-free crystal.

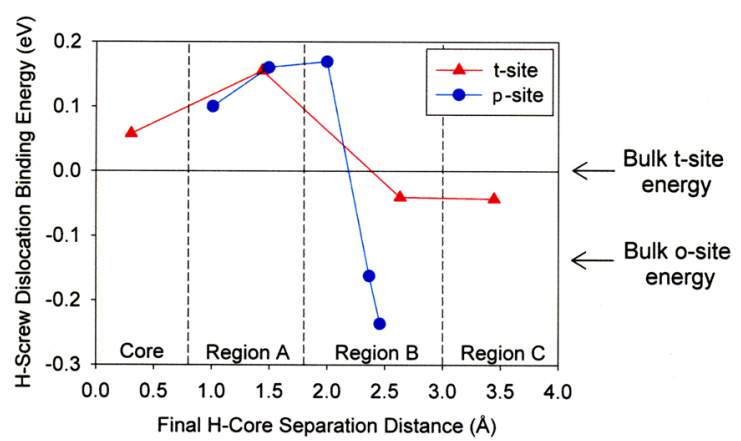

Figure 3. $\mathrm{H}$-screw dislocation binding energies in bcc $\mathrm{Fe}$ as a function of distance from the dislocation core. The tetrahedral sites (t-sites) accommodate a four-fold coordinated $\mathrm{H}$ atom, while the pyramidal sites (p-sites) refer to sites with five-fold coordinated $\mathrm{H}$ atoms. Two-fold octahedral sites (o-sites) are energetically unfavorable.

Because a single vacancy can trap multiple $\mathrm{H}$ atoms $\mathrm{s}^{20,27}$, we investigated incremental binding energies associated with bringing a second $\mathrm{H}$ atom into near-neighbor sites to an existing stable $\mathrm{H}$-screw dislocation defect pair. We found stable $\mathrm{H}-\mathrm{H}$ positions at separation distances in the range 3.5-3.8 $\AA$, but such pairing had minimal effects on the displacement fields of the dislocation core and were characterized by small negative $(-0.01 \mathrm{eV}$ to $-0.03 \mathrm{eV})$ incremental binding energies. Thus, there is little interaction between two $\mathrm{H}$ atoms bound within the core region, and there is no driving force for them to associate in close proximity.

Box 1 summarizes DFT results for binding energies of $\mathrm{H}$-defect interactions discussed in this investigation. There are entries for $\mathrm{H}$-screw dislocation binding for $\mathrm{H}$ occupying either $\mathrm{t}$-sites or p-sites $(0.06-0.17 \mathrm{eV})$; $\mathrm{H}$-vacancy binding $(0.57 \mathrm{eV})$; $\mathrm{H}-\mathrm{C}$ binding $(0.09 \mathrm{eV})$; several $\mathrm{H}$-solute binding energies in three ranges: $0.10-0.25 \mathrm{eV}, 0.01-0.08 \mathrm{eV}$, and essentially zero; and $\mathrm{H}-\mathrm{H}$ binding $(0.04 \mathrm{eV})$.

\section{Discussion and Conclusions}

To present our results and interpretations succinctly, we list and discuss the basis for each conclusion separately, adding information from the literature as needed.

\subsection{The interpretation of Seeger ${ }^{16}$ that the Hcwp is} associated with the motion of $H$ with kinks on non-screw dislocations has been substantiated to the extent that complementary changes occur in the relaxation strengths of the $\alpha$ peak and the Hcwp.

Schoeck ${ }^{28,29}$ has pointed out that experiments utilizing the effects of $\mathrm{H}$ outgassing on the internal friction spectra of deformed $\mathrm{Fe}-\mathrm{H}$ alloys are fruitful because they allow observation
Box 1. Binding Energies for Selected H-Defect Interactions Obtained by Density Functional Theory

\section{H-Screw Dislocation (with distances from central core)}

t-site: $0.06 \mathrm{eV}$ (at $0.3 \AA$ )

t-site: $0.17 \mathrm{eV}$ (at $1.5 \AA$ )

p-site: $0.10 \mathrm{eV}$ (at $1.0 \AA$ )

p-site: $0.17 \mathrm{eV}$ (at $1.5 \AA$ )

p-site: $0.17 \mathrm{eV}$ (at $2.0 \AA$ )

\section{H-Vacancy ${ }^{20}$}

o-site $\mathrm{H}$ displaced $0.2 \AA$ toward vacancy: $0.57 \mathrm{eV}$

$$
\text { H-C }{ }^{20}
$$

t-site H, o-site C: $0.09 \mathrm{eV}$ (at $3.5 \AA$ separation)

\section{H-Solute (all t-site $\mathbf{H})^{20}$}

H- Cd, Mg, Sc, Y: 0.10-0.25 eV

H-Ni, V, Al, Cu, Zn, Nb, Ti: 0.01-0.08 eV

$\mathrm{H}-\mathrm{Co}, \mathrm{Mo}, \mathrm{Cr}, \mathrm{Mn}, \mathrm{Si}: \sim 0.00 \mathrm{eV}$

H-H ${ }^{21}$

t-site $\mathrm{H}$ atoms: $0.04 \mathrm{eV}$

of H-dislocation interaction with minimum changes in the relevant dislocation substructure. Such experiments have been done by Gibala ${ }^{4,9}$, Takita and Sakamoto ${ }^{30}$, and Ritchie et al. ${ }^{31}$. The combined results demonstrate complementary changes in the relaxation strengths of the $\alpha$ peak and the Hcwp: When $\mathrm{H}$ is introduced into bcc $\mathrm{Fe}$, the $\alpha$ peak decreases, those dislocations being pinned by $\mathrm{H}$ atoms, as the Hcwp correspondingly grows; when $\mathrm{H}$ is outgassed, the Hcwp decreases and the $\alpha$ peak recovers toward its original height prior to $\mathrm{H}$ charging. Figure 4 demonstrates these facts.

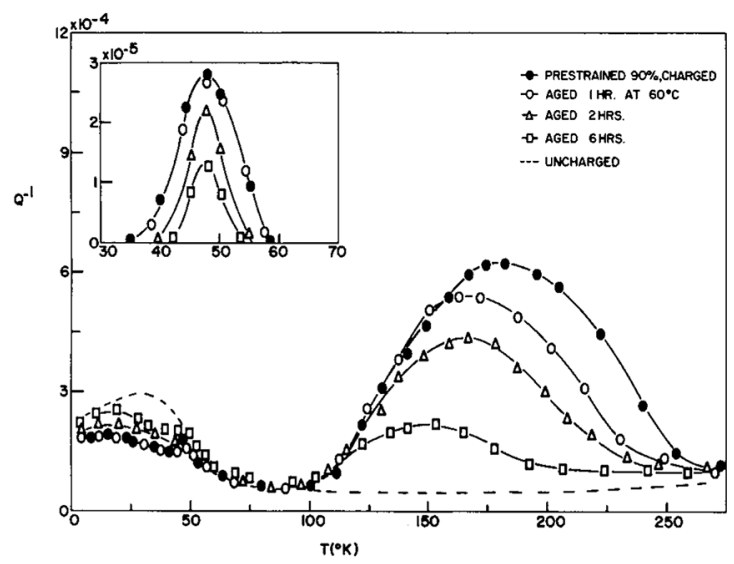

Figure 4. The effect of $\mathrm{H}$ outgassing on the $80 \mathrm{kHz}$ internal friction spectrum of heavily prestrained and $\mathrm{H}$-charged bcc Fe. During $\mathrm{H}$ outgassing, the $\alpha$ peak originally observed at $30 \mathrm{~K}$ in uncharged $\mathrm{Fe}$ recovers in height and temperature, while the Hcwp is correspondingly reduced (from Gibala ${ }^{4,9}$ ).

Figure 4 and other results in the literature also demonstrate that the outgassing-induced reduction in relaxation strength of the Hcwp is larger than the corresponding increase in the $\alpha$ peak, as recognized by Schoeck ${ }^{28,29}$. There are also important 
decreases in the temperature of the Hcwp with increased $\mathrm{H}$ outgassing apparent in Figure 4 as first analyzed by Gibala 9 . These effects offer support for a modified form of a Schoeck model ${ }^{6}$ for the Hcwp discussed in the following section.

\subsection{The solute-dragging theory of the cwp by Schoeck ${ }^{6}$ may have quantitative applicability to the Hcwp if modified to involve H-non- screw dislocation interaction.}

The original theory of Schoeck imagined a generalized solute-laden dislocation bowing string-like between two well-anchored pinning points. It was formulated before the intrinsic origins of the $\alpha$ and $\gamma$ peaks were firmly established. However, the basic mathematical description should be applicable if one of the anchor points is an immobile screw dislocation effectively pinned behind its Peierls barrier, but with limited mobility along its length, and the bowing dislocations are non-screw types.

Thus, the H-dislocation binding energy obtained from internal friction results by Gibala ${ }^{4,9}$ and others likely applies to mixed dislocations, such as $71^{\circ}$ dislocations proposed by Seeger ${ }^{16}$, Fantozzi and Ritchie ${ }^{13}$, and Schultz ${ }^{14}$, but possibly other H-non-screw dislocation complexes as part of the sub-peak structure of the $\alpha$ peak $^{31}$. The changes in Hcwp temperature with $\mathrm{H}$ outgassing shown in Figure 4 fit well with a Schoeck-like model for an average binding energy of $0.3 \mathrm{eV}$ : qualitatively, the more $\mathrm{H}$ atoms at dislocations sites, the more energy required for $\mathrm{H}$ to move with the associated dislocations, resulting in higher peak temperatures.

\subsection{The theory of Hirth ${ }^{3}$ suggests the Hcwp has its origin in $\mathrm{H}$-screw-dislocation binding manifested as the temperature-reduced $\gamma$ peak by H. It has merit in that the implied binding energy of 0.2-0.3 eV is similar to that determined by DFT calculations in this and other investigations. If the Hirth mechanism operates in tandem with the Seeger mechanism, an unmodified Schoeck model could offer an apt description of the Hcwp.}

In his review of effects of $\mathrm{H}$ on mechanical behavior of Fe and steels, Hirth attempted to reconcile many different types of experimental results, including observations of H-enhanced localized plasticity (HELP) ${ }^{17}$ during $\mathrm{H}$ embrittlement, with the internal friction spectra exhibiting the $\alpha, \gamma$ and Hcwp relaxation peaks in bcc Fe. The DFT results in Box 1 and Figure 3 illustrate $\mathrm{H}$-screw dislocation binding energies of sites in the core and near-core regions of a screw dislocation vary substantially, with attractive energies in the range $0.06-0.17 \mathrm{eV}$ for some t-sites and p-sites. The result suggests agreement with Hirth's approach to the HELP mechanism and with Kirchheim's defectantbased support of Hirth's interpretation.
Additional support for Hirth's interpretation comes from quantum-mechanics/molecular-mechanics (QM/ $\mathrm{MM}$ ) calculations of Zhao and $\mathrm{Lu}^{32}$ that employ a smaller computational cell than ours. They determined a H-screw dislocation binding energy of $0.27 \mathrm{eV}$, a value within Hirth's predicted range. Zhao and Lu also determined by QM/MM methods a H-edge dislocation binding energy of $0.47 \mathrm{eV}$, which is addressed in the following section.

A possible difficulty with the Hirth-Kirchheim approach involves changes in the Hcwp peak temperatures portrayed in Figure 4. One would expect the internal friction results to show that more $\mathrm{H}$ atoms at screw dislocations should move the peak temperatures to lower temperatures if a H-softening, HELP-like mechanism is operative in concert with changes in the $\gamma$ peak temperature. However, this is not observed, and none of the changes of the $\gamma$ peak with $\mathrm{H}$ outgassing ${ }^{31}$ appear to correlate with changes in magnitude and temperature of the Hcwp to the extent observed for the $\alpha$ peak $^{4,30,31}$.

In fairness to the Hirth approach, which fits well with many experimental observations outside of internal friction results, effects of interactions of screw dislocations with mobile defects other than $\mathrm{H}$ could mask complementary changes in the Hcwp and the $\gamma$ peak. Taking into account the significant extent to which the Schoeck model also describes the effect of $\mathrm{H}$ outgassing on the Hcwp temperature suggests an intriguing idea: What if both the Seeger mechanism and the Hirth mechanism are operative, more or less in tandem, and the Hcwp temperature is determined by the extent to which $\mathrm{H}$-mixed/edge dislocation and $\mathrm{H}$-screw dislocation interactions "meet" in internal friction-temperature space to allow Schoeck-like bowing to occur? The basic idea is demonstrated schematically by the horizontal arrows in Figure 1. In additional support of such an approach, Miodownik ${ }^{33}$ demonstrated that peak height-peak temperature data from several investigations involving different frequency ranges could be described in terms of the Schoeck model relaxation time and relaxation strength, similar in manner to that presented by Gibala ${ }^{9}$.

The Hcwp's in Figure 4 are very broad relative to the theoretical Debye curve for a single relaxation process. They are characterized by a log normal distribution parameter in the 3-5 range 4 and could contain overlapping Seeger-like and Hirth-like component peaks. Based largely on experiments on single crystals, Weller ${ }^{34}$ has proposed an overlapping twomechanism peak structure for heavy-interstitial-containing systems such as Nb-O, Ta-O, and Fe-N. Similar experiments on $\mathrm{Fe}-\mathrm{H}$ alloys are needed to determine more accurately the extent to which the Hcwp might involve component mechanisms. New experiments that employ specimens with well-characterized dislocation substructures obtained by use of deformation temperature as a systematic variable to alter relative edge/mixed versus screw dislocation distributions will be required to determine the viability of this interpretation. 


\subsection{The large binding energy of $0.6 \mathrm{eV}$ for $H$-vacancy pairs suggests vacancies are a significant component of deep traps experimentally identified by Kumnick and Johnson ${ }^{35}$ and attributed by Hirth ${ }^{3}$ to mixed dislocations and deep-core sites.}

In addition to his suggested $\mathrm{H}$-screw dislocation binding energy of 0.2-0.3 eV, Hirth presented a substantial compilation of other $\mathrm{H}$-trap energies, including an entry for H-mixed dislocation binding of $0.6 \mathrm{eV}$. This assignment is based in part on $\mathrm{H}$ permeation experiments of Kumnick and Johnson, wherein a trapping defect with a binding energy of $\sim 0.6 \mathrm{eV}$ and trap densities in the range $10^{22}-10^{23}$ per $\mathrm{m}^{3}$ in deformed $\mathrm{Fe}$ were determined. At the time, H-vacancy binding was thought to be too small, about $0.4 \mathrm{eV}^{36}$, to account for the permeation lag in deformed materials. However, the DFT results in Box 1, and similar calculations by other investigators ${ }^{27,37}$, show that $\mathrm{H}$-vacancy binding is large, $0.6 \mathrm{eV}$, and that a single vacancy can trap multiple $\mathrm{H}$ atoms.

A simple calculation demonstrates that vacancy trap densities for $\mathrm{H}$ are sufficient to account for the KumnickJohnson results. Assume that each vacancy can trap only one $\mathrm{H}$ atom, $\beta_{\mathrm{H}}=1$ in the equation below. The number of vacancy traps for $\mathrm{H}, \mathrm{N}_{\mathrm{T}}$, is related to the required vacancy concentration $\mathrm{C}_{\mathrm{v}}$ by

$$
N_{T}=\beta_{H} C_{V}\left(N_{o} \rho_{F e} / A_{F e}\right)
$$

where $\mathrm{N}_{\mathrm{o}}$ is Avagadro's number, $\rho_{\mathrm{Fe}}$ is the density of Fe, and $\mathrm{A}_{\mathrm{Fe}}$ is the atomic mass of $\mathrm{Fe}$. For specific trap densities determined by Kumnick and Johnson, $5 \times 10^{22}$ and $2 \times 10^{23}$ $\mathrm{m}^{-3}$ for specimens cold worked $30 \%$ and $80 \%$, respectively, the corresponding values of $\mathrm{C}_{\mathrm{v}}$ are $6 \times 10^{-7}$ and $2 \times 10^{-6}$. These values are well within the range expected for plasticdeformation-produced vacancies ${ }^{38}$, without taking into account additional support from Fukai' ${ }^{39}$ well-documented concept of H-induced 'superabundant' vacancies in metals.

Thus, the following modifications of H-trap binding concepts appear to be required: (1) H-vacancy complexes involving binding energies of the order $0.6 \mathrm{eV}$ (and larger) represent deep traps for $\mathrm{H}$ in bcc $\mathrm{Fe}$ and steels and may play an integral role mechanistically in $\mathrm{H}$ embrittlement, perhaps as suggested by Nagumo and co-workers ${ }^{40}$ and elaborated by Li and co-workers ${ }^{41}$. (2) The H-dislocation binding energy landscape might not extend to the large values given in Hirth's tabulation. Instead, values of binding energies might be: $\mathrm{H}$-screw dislocation, 0.2-0.3 eV; H-mixed dislocation, $\sim 0.3 \mathrm{eV}$; $\mathrm{H}$-edge dislocation, $0.4-0.5 \mathrm{eV}$. Future computational refinements and additional experiments should reduce the uncertainty.
eV determined by DFT suggest that the peaks attributed by Heller ${ }^{42}$, Gibala ${ }^{4}$ and Lord $^{43}$ to a H Snoek peak probably involve H-point defect complexes, as suggested by experiments by Au and Birnbaum ${ }^{44}$ and Dufresne et al. ${ }^{45}$. Additionally, $H$ trapped effectively in solid solution as part of $H$-vacancy complexes might contribute to one or more of the observed relaxations.

Although Heller, Gibala and Lord (HGL) individually interpreted a small relaxation peak they independently observed at temperatures $30 \mathrm{~K}(1 \mathrm{~Hz}), 48 \mathrm{~K}(80 \mathrm{kHz})$ and $120 \mathrm{~K}(10 \mathrm{MHz})$, respectively, as a H Snoek peak, this is probably not the case. These three results as possible $\log$ (frequency)/reciprocal-temperature data points come from Fe materials of different purities and fit imperfectly on an Arrhenius plot, although they suggest an average activation energy $\mathrm{Q}$ and pre-exponential frequency factor $\mathrm{f}_{\mathrm{o}}$ of $0.08 \mathrm{eV}$ and $10^{13} \mathrm{~s}^{-1}$, respectively, in approximate agreement with parameters for $\mathrm{H}$ diffusion in essentially un-trapped $\mathrm{Fe}^{11}$.

Experiments by Dufresne et al. on high purity Fe-H alloys do not exhibit a H Snoek peak nor do they reproduce the Heller version of a H Snoek peak, except possibly as a deformation-related, $\mathrm{H}$-charging artifact that appears to be part of the $\alpha$ peak spectrum. In such high purity Fe, $\mathrm{H}$ occupies tetrahedral sites ${ }^{3}$, as confirmed by DFT calculations. DFT also shows that the addition of vacancies as a pointdefect trap for $\mathrm{H}$ in bcc Fe stabilizes the octahedral sites for $\mathrm{H}$ occupancy ${ }^{20} . \mathrm{H}$ in a nearest-neighbor octahedral site to a vacancy relaxes $0.2 \AA$ toward the vacancy, creating an $<001>$ tetragonal defect in which the $\mathrm{H}$ atom in the defect pair might reorient under an internal friction stress and effect a H Snoek-like relaxation.

At the low peak temperatures involved, $\mathrm{H}$ in highpurity or less-pure Fe can also associate with other point defects, even for small binding energies of the order 0.1 $\mathrm{eV}$ in Box 1, and might cause $\mathrm{H}$-point-defect relaxations of the type observed by HGL ${ }^{45}$. H-based bound-pair defects, whether involving relatively large or small binding energies, can give a quantitative indication of $\mathrm{H}$ present in solid solution and offer insight into the partitioning of $\mathrm{H}$ in various crystal and defect sites.

\section{Acknowledgements}

The authors acknowledge helpful discussions with Dr. Scott Jorgenson. Drs. Christopher Woodward and Dallas Trinkle provided critical insight on use of first-principles Green's function flexible boundary conditions methodology. Funding from the General Motors Corporation and the US Department of Energy is also acknowledged.

\subsection{Small H-solute binding energies of $\sim 0.1$}




\section{References}

1. Snoek JL. Effect of small quantities of carbon and nitrogen on the elastic and plastic properties of iron. Physica. 1941;8(7):711-733.

2. Köster W, Bangert L, Hahn R. Das Dämpfungsverhalten von gerecktem technischem eisen. Archiv für das Eisenhüttenwesen. 1954;25(11/12):569-578.

3. Hirth JP. Effects of hydrogen on the properties of iron and steel. Metallurgical Transactions A. 1980;11(6):861-890.

4. Gibala R. Internal Friction in Hydrogen-Charged Iron. Transactions of the Metallurgical Society of AIME. 1967;239(10):1574-1585.

5. Sturges CM, Miodownik AP. The interaction of hydrogen and dislocations in iron. Acta Metallurgica. 1969;17(9):1197-1207.

6. Schoeck G. Friccion interna debido a la interaction entre dislocaciones y atomos solutos. Acta Metallurgica. 1963;11(6):617-622.

7. Kamber K, Keefer D, Wert C. Interaction of interstitials with dislocations in iron. Acta Metallurgica. 1961;9(5):403-414.

8. Boone DH, Wert CA. Interstitial-Dislocation Interaction in Niobium. Journal of the Physical Society of Japan. 1963;18(Suppl. 1):141-148.

9. Gibala R. Comments on "the cold-work damping peak in alpha-iron". Scripta Metallurgica. 1968;2(1):13-16.

10. Keh AS. Dislocation Arrangement in Alpha Iron During Deformation and Recovery. In: Newkirk JB, Wernick JH, eds. Direct Observation of Imperfections in Crystals. New York: Interscience; 1962. p. 213-281.

11. Oriani RA. The diffusion and trapping of hydrogen in steel. Acta Metallurgica. 1970;18(1):147-157.

12. Chambers RH. Dislocation Relaxations in Body-Centered Cubic Transition Metals. In: Mason WP, ed. Physical Acoustics - Principles and Methods. Volume III-A. New York: Academic Press; 1966. p. 123-197.

13. Fantozzi G, Ritchie IG. Internal Friction Caused by the Intrinsic Properties of Dislocations. Journal de Physique Colloques. 1981;42(C5):3-23.

14. Schultz H. Defect parameters of b.c.c. metals: group-specific trends. Materials Science and Engineering: A. 1991;141(2):149-167.

15. Benoit W. Dislocation-Lattice Interactions. In: Schaller R, Fantozzi G, Gremaud G, eds. Mechanical Spectroscopy $Q^{-1}$ 2001 with Applications to Materials Science. Zürich: Trans Tech Publications; 2001. p. 158-177.

16. Seeger A. A theory of the Snoek-Köster relaxation (cold-work peak) in metals. Physica Status Solidi (a). 1979;55(2):457-468.

17. Robertson IM, Sofronis P, Nagao A, Martin ML, Wang S, Gross DW, et al. Hydrogen Embrittlement Understood. Metallurgical and Materials Transactions A. 2015;46(6):2323-2341.

18. Gibala R, Kumnick AJ. Hydrogen Trapping in Iron and Steels. In: Gibala R, Hehemann RF, eds. Hydrogen Embrittlement and Stress Corrosion Cracking. Metals Park: American Society for Metals; 1984. p. 61-77.

19. Kirchheim R. Solid solution softening and hardening by mobile solute atoms with special focus on hydrogen. Scripta Materialia. 2012;67(9):767-770.
20. Counts WA, Wolverton C, Gibala R. First-principles energetics of hydrogen traps in $\alpha$-Fe: Point defects. Acta Materialia. 2010;58(14):4730-4741.

21. Counts WA, Wolverton C, Gibala R. Binding of multiple $\mathrm{H}$ atoms to solute atoms in bcc Fe using first principles. Acta Materialia. 2011;59(14):5812-5820.

22. Woodward C, Rao SI. Flexible Ab initio boundary conditions: simulating isolated dislocations in bcc Mo and Ta. Physical Review Letters. 2002;88(21):216402.

23. Woodward C, Rao SI. Ab-initio simulation of isolated screw dislocations in bec Mo and Ta. Philosophical Magazine A. 2001;81(5):1305-1316.

24. Sinclair JE, Gehlen PC, Hoagland RG, Hirth JP. Flexible boundary conditions and nonlinear geometric effects in atomic dislocation modeling. Journal of Applied Physics. 1978;49(7):3890-3897.

25. Rao S, Hernandez C, Simmons JP, Partharasarthy TA, Woodward C. Green's function boundary conditions in two-dimensional and three-dimensional atomistic simulations of dislocations. Philosophical Magazine A. 1998;77(1):231-256.

26. Ito K, Vitek V. Atomistic study of the non-Schmid effects in the plastic yielding of bcc metals. Philosophical Magazine A. 2001;81(5):1387-1407.

27. Tateyama Y, Ohno T. Stability and clusterization of hydrogenvacancy complexes in $\alpha$-Fe: An ab-initio study. Physical Review B. 2003;67(17):174105

28. Schoeck G. The cold work peak. Scripta Metallurgica. 1982;16(3):233-239.

29. Schoeck G. On the mechanism of the Snoek-Koester relaxation. Scripta Metallurgica. 1988;22(3):389-394.

30. Takita K, Sakamoto K. Low temperature internal friction peak and hydrogen cold-work peak in deformed $\alpha$-iron. Scripta Metallurgica. 1976;10(5):399-403.

31. Ritchie IG, Dufresne JF, Moser P. Internal friction in deformed hydrogen-doped iron. Physica Status Solidi (a). 1979;52(1):331340 .

32. Zhao Y, Lu G. QM/MM study of dislocation-hydrogen/helium interactions in $\alpha$-Fe. Modelling and Simulation in Materials Science and Engineering. 2011;19(6):065004.

33. Miodownik AP. The Interaction of Hydrogen with Dislocations, Stacking Faults, and Other Interfaces. In: Staehle RW, Hochmann J, McCright RD, Slater JE, eds. Stress Corrosion Cracking and Hydrogen Embrittlement of Iron Base Alloys. Houston: National Association of Corrosion Engineers; 1977. p. 272-285.

34. Weller M. The Snoek-Köster Relaxation in Body-Centered Cubic Metals. Journal de Physique Colloques. 1983;44(C9):63-82.

35. Kumnick AJ, Johnson HH. Deep trapping states for hydrogen in deformed iron. Acta Metallurgica. 1980;28(1):33-39.

36. DeCooman BC, Speer JG. Fundamentals of Steel Product Physical Metallurgy. Warrendale: AISI; 2012. p. 45.

37. Mirzaev DA, Mirzoev AA, Okishev KY, Verkhovykh AV. Hydrogen-vacancy interaction in bcc iron: ab initio calculations and thermodynamics. Molecular Physics. 2014;112(13):17451754. 
38. Detemple K, Kanert O, DeHossen JT, Murty KL. In-situ nuclear magnetic resonance investigation of deformation-generated vacancies in aluminum. Physical Review B. 1995;52(1):125-133.

39. Fukai Y. Superabundant Vacancies Formed in Metal-Hydrogen Alloys. Physica Scripta. 2003;T103(1):11-14.

40. Takai K, Shoda H, Suzuki H, Nagumo M. Lattice defects dominating hydrogen-related failure of metals. Acta Materialia. 2008;56(18):5158-5167.

41. Neeraj T, Srinivasan R, Li J. Hydrogen embrittlement of ferritic steels: Observations on deformation microstructure, nanoscale dimples and failure by nanovoiding. Acta Materialia. 2012;60(13-14):5160-5171.
42. Heller WR. Quantum effects in diffusion: internal friction due to hydrogen and deuterium dissolved in $\alpha$-iron. Acta Metallurgica. 1961;9(6):600-613.

43. Lord AE Jr. Diffusion of hydrogen in $\alpha$-iron at about $120^{\circ} \mathrm{K}$. Acta Metallurgica. 1967;15(7):1241-1244.

44. Au JJ, Birnbaum HK. Magnetic relaxation studies of the motion of hydrogen and deuterium in iron. Acta Metallurgica. 1978;26(7):1105-1116.

45. Dufresne JF, Seeger A, Groh P, Moser P. Hydrogen relaxation in $\alpha$-iron. Physica Status Solidi (a). 1976;36(2):579-589. 